\title{
Evaluación de la continuidad del cuidado brindado por médicos de atención primaria en un seguro de salud de un hospital universitario privado de Argentina
}

\author{
Evaluation of the continuity of care provided by primary care physicians in a private university hospital's health
}

insurance scheme in Argentina

Gabriel Esteban Villalón $^{a}$ (D), Karin Kopitowski ${ }^{\mathrm{a}}$ (D), Sergio Adrian Terrasa ${ }^{\mathrm{a}}$ (D), Santiago Esteban ${ }^{\mathrm{a}}$, María Victoria Ruiz Yanzi $^{\mathrm{a}}$ (D)

\begin{abstract}
Resumen
Antecedentes. La continuidad del cuidado ( $\mathrm{CoC}$ ) suele describir en qué medida los pacientes ven al mismo profesional a lo largo del tiempo. Constituye un elemento necesario para garantizar la longitudinalidad en la atención, elemento clave de la atención primaria y de la medicina familiar en particular, entendido como el cuidado centrado en la persona a lo largo del tiempo, independientemente del tipo de problema consultado. Esta ha sido vinculada con una serie de beneficios para los pacientes y algunos indicadores duros de utilización de servicios de salud, por ejemplo visitas a la central de emergencias (CE), internaciones hospitalarias y mortalidad. En Argentina y América Latina no habíamos podido identificar estudios que dieran cuenta del nivel local de CoC o de su impacto en la utilización de los servicios de salud.

Objetivo. Cuantificar los niveles de CoC de los afiliados de un Seguro de Salud de un Hospital Universitario Privado y describir su asociación con la utilización de servicios de salud, tales como consultas a la CE e internaciones hospitalarias. Métodos. Estudio observacional, descriptivo, de cohorte retrospectiva; realizado sobre la totalidad de afiliados que hubieran realizado al menos dos consultas programadas con un médico de atención primaria entre 2015 y 2016.

Las mediciones principales consistieron dos indicadores: el índice del proveedor usual de cuidados y el índice de continuidad del cuidado (UPC y COC, respectivamente, por sus iniciales en inglés) -cuyos valores oscilan entre 0 y 1 , y se interpretan como: el paciente siempre fue atendido por diferentes médicos vs siempre por el mismo médico-y el recuento de visitas a la CE e internaciones hospitalarias.

Resultados. Fueron identificados 112.062 pacientes con una mediana de edad de $59\left(\mathrm{P}_{5}-\mathrm{P}_{95}, 4\right.$ a 87$)$ años. Las medianas $\left(P_{5}-P_{95}\right)$ de los indicadores de continuidad, UPC y COC, fueron $0,66(0,25$ a 1$)$ y $0,33(0,04$ a 1$)$, respectivamente. Se observó una asociación inversa y estadísticamente significativa entre los indicadores de $\mathrm{CoC}$ y la cantidad de visitas a la $\mathrm{CE}$, evidenciada por una razón de incidencia decreciente de consultas contra quintilos crecientes de COC (tomando como referencia el quintilo más bajo de continuidad). Esta asociación no pudo demostrarse para las internaciones.

Conclusiones. Los niveles de CoC alcanzados y su impacto en la utilización de servicios de salud reflejan valores semejantes a los de otros estudios publicados fuera de nuestra región y podrían aportar elementos útiles para delinear estrategias tendientes a la mejora de la calidad de la atención médica.
\end{abstract}

\begin{abstract}
Background. Continuity of care $(\mathrm{CoC})$ usually describes the extent to which patients see the same professional over time. It is a necessary element to ensure continuity of care, a key element of primary care and family medicine, in particular, understood as person-centred care over time, regardless of the type of problem consulted.

It has been linked to a series of benefits for patients as well as to hard indicators of health services utilization (e.g. emergency room [ER] visits, hospitalizations) and mortality. In Argentina and Latin America, we have not been able to identify studies that account for the local level of $\mathrm{CoC}$ and its impact.

Objective. To quantify the $\mathrm{CoC}$ levels among members of a private university hospital's health insurance scheme and to describe its association with the use of health services, such as ER visits and hospitalizations.

Methods. Observational, descriptive, retrospective cohort study; conducted on the total number of members who had made at least two schedurivled consultations with a primary care physician between 2015 and 2016 .

The main outcomes were two indicators: the usual provider continuity index and the continuity of care index (UPC and COC, respectively) -both range between 0 and 1, and are interpreted as: the patient was always treated by different doctors vs. always by the same doctor- and the number of visits to the ER and hospitalizations.

Results. A total of 112,062 patients with a median age of $59\left(P_{5}-P_{95}, 4\right.$ to 87$)$ years were identified.

The median $\left(\mathrm{P}_{5}-\mathrm{P}_{95}\right)$ of the continuity indicators, UPC and COC, was $0.66(0.25$ to 1$)$ and $0.33(0.04$ to 1$)$, respectively. A significant inverse association was observed between the $\mathrm{CoC}$ indicators and the number of ER visits, evidenced by a decreasing incidence rate of consultations versus increasing quintiles of $\mathrm{CoC}$ (taking the lowest quintile of continuity as a reference). This association was not observed for hospitalizations.

Conclusions. The levels of $\mathrm{CoC}$ reached and their impact on the use of health services reflect similar values to those of other studies published outside our region and could provide useful elements for outlining strategies aimed at improving the quality of medical care.
\end{abstract}

Palabras clave: Continuidad de la Atención al Paciente, Atención Primaria de Salud, Calidad de la Atención de Salud, Longitudinalidad. Keywords: Continuity of Patient Care, Primary Health Care, Quality of Health Care, Longitudinality.

Villalon GE, Kopitowski K, Terrasa SA, Esteban S, Ruiz Yanzi MV. Evaluación de la continuidad del cuidado brindado por médicos de atención primaria 
en un seguro de salud de un hospital universitario privado de Argentina. Evid Actual Pract Ambul. 2021;24(1):e002113.

\section{Introducción}

El concepto de continuidad del cuidado ( $\mathrm{CoC}$ ) suele utilizarse de manera genérica para describir en qué medida los pacientes consultan al mismo profesional a lo largo del tiempo. De manera más específica, se han propuesto diferentes definiciones ${ }^{1-3}$ que podrían resumirse en la siguiente: la $\mathrm{CoC}$ es una característica del proceso de atención de un usuario del sistema de salud en el que la dinámica de las interacciones entre el paciente y los prestadores, y/o la calidad de la conexión y retroalimentación de los eventos que se suceden influyen en la experiencia final de sentirse cuidado ${ }^{4}$

La CoC se convierte asi en un indicador relevante a la atención primaria de la salud (APS), en tanto que es un elemento necesario para garantizar la longitudinalidad en la atención de los problemas de salud de los pacientes ${ }^{5}$.

Este indicador, extensamente estudiado durante las últimas décadas, ha sido desglosado en varios subdominios ${ }^{1,2,6}$, y se ha vinculado con diferentes resultados con impacto sanitario beneficioso, incluyendo desenlaces duros como la reducción de visitas a la central de emergencias (CE), las internaciones e incluso, la mortalidad general ${ }^{7-14}$. Sin embargo, en Argentina y América Latina no habíamos podido identificar estudios que dieran cuenta del nivel local de CoC o de su impacto en la utilización de los servicios de salud.

El Hospital Italiano de Buenos Aires (HIBA) ${ }^{15}$ es un efector privado que brinda asistencia sanitaria a alrededor de 160.000 afiliados del Plan de Salud (PS) que habitan predominantemente en el Área Metropolitana de Buenos Aires. Su infraestructura incluye dos centros hospitalarios de alta complejidad, 19 centros ambulatorios propios, farmacias y consultorios odontológicos, mas una red de prestadores de servicios (tercerizados mediante convenios), localizados en el interior del país con los que el sistema atiende asimismo a sus afiliados en tránsito por otras ciudades.

EI PS brinda asistencia a sus afiliados mediante el modelo de "médico de cabecera" (MC), el cual asigna un médico de atención primaria ([MAP], clínico, médico de familia o pediatra) a cada afiliado para ser el referente y consultor de los problemas de salud más frecuentes.

Los MC ejecutan su práctica mediante la atención de turnos programados (de 20 minutos de duración), sobreturnos (sin tiempo específico asignado e intercalados entre las consultas programadas) y atención de consultas a distancia mediante un sistema de mensajería electrónico, vehiculizado a través de la historia clínica electrónica (HCE).

EI MC es elegido libremente por el afiliado y puede ser cambiado en cualquier momento del proceso de atención, mediante la simple solicitud de un turno programado con un MAP diferente.

La atención primaria ambulatoria se complementa con servicios de consulta no programada, también llamados consultorios de demanda espontanea, que asisten a los afiliados con motivos de consulta urgentes de baja complejidad. Las consultas urgentes de mayor complejidad son asistidas en la CE. Los pacientes que lo requieren son asistidos también a través de internaciones hospitalarias.

Asimismo los afiliados del PS pueden acceder libremente a consultas programadas con médicos especialistas, aunque el sistema estimula la interconsulta guiada por criterio del MC estableciendo un pago adicional por consulta realizada al especialista cuando ésta se realiza sin el aval del MC (gestionado mediante un sistema electrónico de derivación a través de la HCE).
De esta manera, la continuidad en el proceso de atención ambulatoria puede verse alterada cuando el afiliado, por diferentes motivos (ausencia del MC, demora en el acceso a los turnos, decisión del afiliado, etc.) consulta a un MAP diferente del actual MC, sea a través de un turno programado, una consulta de demanda espontánea, consultas a médicos especialistas (sin mediar interconsulta) o consultas a la CE en los horarios o por razones en las que su MC podría haberlo asistido.

El presente estudio se propuso como objetivos: a) cuantificar y describir la continuidad longitudinal del cuidado (CoC) de los afiliados del PS del HIBA, y b) describir la asociación entre dos indicadores de continuidad longitudinal del cuidado y la utilización de servicios de salud como consultas a la CE e internaciones hospitalarias.

\section{Métodos}

\section{Diseño}

Estudio observacional, descriptivo, de cohorte retrospectiva, realizado en base a datos administrativos registrados en una historia clínica electrónica.

\section{Población}

Afiliados al PS del HIBA de cualquier edad que hayan realizado entre el 01 de enero de 2015 y el 31 de diciembre de 2016 al menos dos consultas programadas con un MAP (médico de familia, clínico o pediatra).

\section{Medición de resultados principales}

Para cada paciente incluido en la muestra se construyeron y cuantificaron los índices de continuidad más conmunmente utilizados, el índice del proveedor usual de cuidados (UPC, por sus iniciales en inglés) y el índice de continuidad del cuidado (COC, por sus iniciales en inglés). Para ambos, los valores posibles oscilan entre 0 y 1 , siendo 0 equivalente al cuidado más fragmentado posible y 1 , al cuidado con mayor continuidad posible. En una revisión narrativa publicada en este mismo número de esta revista se describen con mayor detalle su construcción y sus características ${ }^{4}$

Para el índice UPC se tomó como "médico usual" aquel médico que hubiera asistido con mayor frecuencia al paciente en cuestión (frente a un eventual empate, se asumió como médico usual aquel que brindó la última asistencia). Para cada indicador, a su vez, fueron exploradas dos variantes (UPC o UPC ampliado [UPCa] y $\mathrm{COC}$ o $\mathrm{COC}$ ampliado [COCa]), según incorporen en su construcción solo las consultas que tuvieron lugar en el marco de turnos programados con un médico de cabecera, o que incluyan además aquellas que ocurrieron en los consultorios de atención no programada o de demanda espontánea.

En la construcción de estos indicadores no fueron incluidas las consultas domiciliarias realizadas por los MAP, ni las realizadas en forma remota por teléfono o través de alguna plataforma virtual (durante el período analizado, previo a la pandemia por COVID-19, éstas últimas representaban una proporción muy baja del total de las consultas). Tampoco fueron incluidas las consultas de atención primaria prestadas por ginecólogos, oftalmólogos, enfermeros u otros profesionales de la salud no médicos.

Para medir la utilización de los servicios de emergencias médicas o internaciones hospitalarias, se contabilizó para cada paciente incluido en la muestra la sumatoria de visitas (o episodios) a dichos ámbitos, durante el año siguiente (01/01/2017 al

\footnotetext{
a Servicio de Medicina Familiar y Comunitaria, Hospital Italiano de Buenos Alres, Argentina. gabriel.villalon@hospitalitaliano.org.ar, karin.kopitowski@hospitalitaliano.org.ar, sergio.terrasa@hospitalitaliano.org.ar, santiago.esteban@hospitalitaliano.org.ar, mariav.ruiz@hospitalitaliano.org.ar
} 
31/12/2017) al periodo utilizado para la construcción de los indicadores de continuidad del cuidado.

\section{Obtención de los datos}

Fue solicitado al Departamento de Informática en Salud del HIBA el detalle de la totalidad de las consultas contempladas en los criterios de inclusión. Se consideró consulta realizada a aquella en la cual los registros informáticos documentaron al paciente como presente y atendido. Se registraron asimismo el recuento de todas las consultas ambulatorias realizadas con médicos especialistas, consultas a la CE, y recuento de episodios de internación hospitalaria.

\section{Análisis estadístico}

Las variables continuas se describieron calculando la media y desvío estándar (DE) o la mediana y los percentilos 25 Y $75\left(\mathrm{P}_{25^{-}}\right.$ $\mathrm{P}_{75}$ ), según su distribución; mientras que las variables categóricas se reportaron como proporciones. Las medidas de asociación se calcularon utilizando el modelo de regresión binomial negativa (ajustando por edad y sexo) y se describieron como razón de incidencia. Para el análisis de los datos se utilizó Stata (versión 13.0).

\section{Consideraciones éticas}

El protocolo de investigación fue aprobado por el Comité de Ética de Protocolos de Investigación del HIBA.

\section{Resultados}

Fueron identificados 112.081 pacientes con una mediana de edad $\left(\mathrm{P}_{5}-\mathrm{P}_{95}\right)$ de 58 (4 a 87) años. La muestra incluyó un mayor porcentaje de mujeres y población adulta (verTabla 1).

En promedio, fueron más frecuentes las consultas al MC por sobre las consultas a la demanda espontánea (media 4,90; DE 3,36 vs. 2,54; DE 3,97), y a su vez se observó una frecuencia similar de consultas entre los MAP (médico de cabecera y demanda espontánea) por sobre las consultas a especialistas (media 7,44; DE 5,76 vs media 8,0; DE 9,29) (verTabla 2).

Las medianas $\left(\mathrm{P}_{5}-\mathrm{P}_{95}\right)$, de los indicadores de continuidad UPC y COC se situaron en $1(0,5$ a 1$)$ y $1(0,33$ a 1$)$, respectivamente, lo cual refleja la escasa propensión de los pacientes a cambiar la figura del médico de cabecera; mientras que al considerar en el indicador las consultas realizadas en los consultorios de demanda espontánea, estos indicadores, UPCa y $\mathrm{COCa}$, fueron menores: $0,66(0,25$ a 1$)$ y $0,33(0,04$ a 1$)$, respectivamente (verTabla 3).

Se observó una asociación inversa y estadísticamente significativa entre los indicadores de $\mathrm{CoC}$ y la cantidad de visitas a la $\mathrm{CE}$, evidenciada por una razón de incidencia decreciente de consultas contra quintilos crecientes de $\mathrm{CoC}$, tomando como referencia el quintilo más bajo de continuidad (ver Tabla 4). Esta asociación no puedo demostrarse para las internaciones (ver Tabla 5).

\section{Discusión}

El presente trabajo surgió inicialmente por la necesidad de incorporar uno o más indicadores validados y relevantes a la APS dentro de un programa de mejora de la calidad de atención médi$\mathrm{ca}^{16}$. Para ello, resultaba indispensable construir dichos indicadores partiendo de datos disponibles y accesibles (en este caso, bases de datos administrativas) ${ }^{17,18}$, explorar su distribución en la población y validar su potencial influencia respecto de resultados con impacto sanitario/asistencial, de manera similar a la comunicada en estudios previos ${ }^{19-23}$.

La decisión de utilizar datos administrativos condicionó que el dominio medido haya sido la continuidad longitudinal de la relación; y esto, a su vez, determinó que fueran elegidos dos de los indicadores más utilizados en la bibliografía ${ }^{18}$ : uno de concentración (el indicador del proveedor usual de cuidados, o UPC por sus iniciales en inglés), y otro de dispersión (el indicador de continuidad del cuidado de Bice y Boxerman, o COC por sus iniciales en inglés) ${ }^{4}$.

En la construcción de ambos indicadores es importante que se tomen en cuenta solamente las consultas realizadas a proveedores que puedan brindar un espectro de cuidados técnicamente similar y en un contexto relacionado ${ }^{6}$. Por este motivo, el universo de consultas incluyó, para cada paciente, a cualquier MAP (médico clínico, de familia o pediatra) que durante el período analizado haya estado disponible en el marco de consultorios de atención programada (incluyendo, asimismo, las consultas que pudieran ocurrir como sobreturnos). Sin embargo, por las características de nuestro sistema de atención, resultó razonable incluir una variante para ambos indicadores (UPCa y COCa) que incluyese también las consultas realizadas en el ámbito de los consultorios de atención no programada (consultorios en el que se atiende por orden de llegada, sin turno previo, en el que el paciente no puede elegir al profesional que lo asiste y en el que, generalmente, se asisten consultas de baja a mediana complejidad).

Consideramos que en este trabajo, los indicadores UPCa y COCa reflejan con mayor fidelidad el valor real de $\mathrm{CoC}$ alcanzado (valores de mediana $\left[\mathrm{P}_{5}-\mathrm{P}_{95}\right]$ de 0,66 [0,44 a 0,83], y 0,33 [0,16 a 0,66 ] para UPCa y COCa, respectivamente) que se asemejan, a su vez, a los valores reportados por otros estudios ${ }^{21-24}$. No obstante, resulta interesante observar el altísimo grado de fidelidad registrado para UPC y COC; es decir, cuando solo se toman en cuenta las consultas que ocurren en los consultorios de atención programada realizadas por médicos que cumplen con el rol de médicos de cabecera. Esto probablemente refleja la posibilidad de libre elección del profesional por parte de los afiliados.

Respecto de la utilización de servicios de emergencias y las internaciones, los resultados obtenidos son acordes a los comunicados por otros estudios ${ }^{20-23}$. En el presente trabajo documentamos una reducción creciente en el número de consultas a la CE a medida que se incrementó el nivel de CoC por quintilo (a mayor quintilo, mayor $\mathrm{CoC}$ ). Esto no fue evidente para las internaciones.

Entre las fortalezas de la investigación se encuentran el gran número de pacientes incluidos (112.081), afiliados a un sistema cerrado de atención, lo que limita pero no excluye la posibilidad de subregistro de consultas, la obtención de datos administrativos a partir de un registro informatizado centralizado, y la medición de las consultas a la CE y las internaciones durante un periodo posterior (en este caso, un año) al tomado para la construcción de los indicadores de CoC (dos años previos).

Entre las debilidades de este trabajo, se encuentran la ausencia de otros indicadores accesorios de CoC (por ejemplo: indicador modificado de continuidad del cuidado [siglas en inglés, $\mathrm{MMCl}$ ], indicador de continuidad secuencial [SECON], indicador integrado de continuidad del cuidado [COCl], entre otros); la carencia de medidas de ajuste -como por ejemplo, por comorbilidad o por desenlaces vinculados a problemas de salud sensibles a la atención primaria; y por último, la dificultad para extrapolar los resultados a otras poblaciones con diferentes sistemas de cobertura médica, dada la gran fragmentación del sistema de salud de Argentina, y/o de diferentes estratos socioeconómicos.

A nuestro entender, el presente estudio representa uno de los pocos publicados en nuestra región sobre esta temática. Consideramos que sus resultados pueden proveer un marco de comparación para otros estudios similares que al menos busquen contrastar el nivel de $\mathrm{CoC}$ de otras poblaciones de la región, o bien, servir de inspiración para refinar el desarrollo de indicadores relacionados aplicables a la mejora de la calidad de atención asistencial. 


\section{Conclusiones}

Los niveles de CoC alcanzados y su impacto en la utilización de servicios de salud reflejan valores semejantes a los de otros estudios publicados fuera de nuestra región, y podrían aportar elementos útiles para delinear estrategias tendientes a la mejora de la calidad de la atención médica.

Conflicto de interés de los autores: Los autores no declaran conflictos de interés.

Fuente de financiamiento: El presente estudio contó con el apoyo de la Fundación MF, ámbito en el que se desempeñan los autores de este trabajo.
Tabla 1. Características demográficas de los pacientes incluidos en la muestra.

\begin{tabular}{|l|l|}
\hline Variable & Resultado \\
\hline Total, $\mathrm{n}$ & 112.081 \\
\hline Edad & \\
$<18 \mathrm{n}(\%)$ & $18.021(16,08)$ \\
$18 \mathrm{a} 65, \mathrm{n}(\%)$ & $47.598(42,47)$ \\
$>65, \mathrm{n}(\%)$ & $46.462(41,45)$ \\
\hline Sexo & \\
Masculino, $\mathrm{n}(\%)$ & $42.323(37,76)$ \\
Femenino, $\mathrm{n}(\%)$ & $69.758(62,24)$ \\
\hline
\end{tabular}

Tabla 2. Número de consultas realizadas entre 2015 y 2016 (dos años), según el ámbito de atención de los pacientes incluidos en la muestra. Notas: *No incluye consultas de acceso directo, como oftalmología y ginecología. **En la sede central del Hospital Italiano de Buenos Aires la central de emergencias pediátrica incluye la función de demanda espontánea de consultas pediátricas de baja complejidad. DE: desvío estandar. P25-P75: percentilos 25 y 75.

\begin{tabular}{|c|c|c|c|c|c|}
\hline & $\begin{array}{l}\text { Médico de } \\
\text { cabecera } \\
\text { Mediana } \\
\text { (P25-P75) }\end{array}$ & $\begin{array}{c}\text { Consultorio de } \\
\text { demanda } \\
\text { espontanea } \\
\text { Mediana (P25-P75) }\end{array}$ & $\begin{array}{c}\text { Consultorio de } \\
\text { especialistas* }^{*} \\
\text { Mediana (P25-P75) }\end{array}$ & $\begin{array}{c}\text { Central de } \\
\text { emergencias } \\
\text { Media } \pm \text { DE }\end{array}$ & $\begin{array}{c}\text { Internaciones } \\
\text { hospitalarias } \\
\text { Media } \pm \mathrm{DE}\end{array}$ \\
\hline Total & $4(3 \mathrm{a} 6)$ & $1(0$ a 3$)$ & $5(2$ a 11) & $1,46 \pm 3,14$ & $0,23 \pm 0,76$ \\
\hline \multicolumn{6}{|l|}{ Sexo } \\
\hline Masculino & $4(2$ a 6$)$ & 1 (0 a 3) & $4(1$ a 10$)$ & $1,73 \pm 3,61$ & $0,24 \pm 0,79$ \\
\hline Femenino & 4 (3 a 6) & $1(0$ a 4$)$ & $5(2$ a 12) & $1,30 \pm 2,82$ & $0,22 \pm 0,74$ \\
\hline \multicolumn{6}{|l|}{ Edad } \\
\hline$<18$ & $4(3$ a 6$)$ & 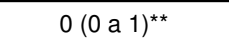 & $2(0$ a 4$)$ & $4,85 \pm 5,7^{* *}$ & $0,20 \pm 0,58$ \\
\hline 18 a 65 & 3 (2 a 5) & $1(0$ a 3$)$ & $4(1$ a 8) & $0,83 \pm 1,87$ & $0,14 \pm 0,61$ \\
\hline$>65$ & $5(3$ a 8$)$ & $2(0$ a 5$)$ & $9(4$ a 17$)$ & $0,79 \pm 1,58$ & $0,33 \pm 0,93$ \\
\hline
\end{tabular}

Tabla 3. Indicadores de continuidad del cuidado de los pacientes incluidos en la muestra. Notas: P25-P75: percentilos 25 y 75.

\begin{tabular}{|c|c|c|c|c|}
\hline Indicadores & $\begin{array}{c}\text { UPC } \\
\text { Mediana (P25-P75) } \\
\end{array}$ & $\begin{array}{l}\text { UPCa } \\
\text { Mediana (P25-P75) }\end{array}$ & $\begin{array}{l}\text { COC } \\
\text { Mediana (P25-P75) } \\
\end{array}$ & $\begin{array}{l}\text { COCa } \\
\text { Mediana (P25-P75) }\end{array}$ \\
\hline Total & $1(1 \mathrm{a} 1)$ & $0,66(0,44$ a 0,83$)$ & 1 (1 a 1) & $0,33(0,16$ a 0,66$)$ \\
\hline \multicolumn{5}{|l|}{ Sexo } \\
\hline Masculino & 1 (1 a 1) & $0,66(0,46$ a 0,85$)$ & 1 (1 a 1) & $0,35(0,16$ a 0,71$)$ \\
\hline Femenino & 1 (1 a 1) & $0,63(0,44$ a 0,83$)$ & 1 (1 a 1) & $0,33(0,16$ a 0,66$)$ \\
\hline \multicolumn{5}{|l|}{ Edad } \\
\hline$<18$ & 1 (1 a 1) & $0,62(0,43$ a 0,83$)$ & 1 (1 a 1) & $0,33(0,16$ a 0,66$)$ \\
\hline 18 a 65 & 1 (1 a 1) & $0,63(0,42$ a 0,83$)$ & 1 (1 a 1) & $0,33(0,16$ a 0,66$)$ \\
\hline$>65$ & 1 (1 a 1$)$ & $0,66(0,48$ a 0,83$)$ & 1 (1 a 1$)$ & $0,40(0,21$ a 0,68$)$ \\
\hline
\end{tabular}


Tabla 4. Asociación entre la continuidad del cuidado (por quintilos de UPCa y COCa) y el número de visitas a central de emergencias durante 2017 (al año siguiente). Notas: *Ajustado por edad y sexo. DE: desvío estandar; IC 95\%: intervalo de confianza del $95 \%$; PRI: Razón de incidencia (en inglés Incidence Rate Ratio). NA: no aplica (valor de referencia)

\begin{tabular}{|c|c|c|c|c|}
\hline Quintilo & $\begin{array}{c}\text { Consultas a CE por } \\
\text { quintilo de UPCa, Media } \\
\text { (DE) }\end{array}$ & $\begin{array}{c}\text { Consultas a CE por } \\
\text { quintilo de COCa, Media } \\
\text { (DE) }\end{array}$ & UPCa, RI (IC $95 \%)^{*}$ & COCa, RI (IC $95 \%)^{*}$ \\
\hline 1 & $0,90(1,93)$ & $0,90(1,94)$ & NA & NA \\
\hline 2 & $0,74(1,70)$ & $0,73(1,67)$ & $0,85(0,81$ a 0,88$)$ & $0,88(0,84$ a 0,91$)$ \\
\hline 3 & $0,63(1,54)$ & $0,63(1,56)$ & $0,73(0,70$ a 0,76$)$ & $0,77(0,74$ a 0,80$)$ \\
\hline 4 & $0,59(1,46)$ & $0,59(1,47)$ & $0,73(0,70$ a 0,76$)$ & $0,76(0,73$ a 0,79$)$ \\
\hline
\end{tabular}

Tabla 5. Asociación entre la continuidad del cuidado (por quintilos de UPCa yCOCa) y el número de internaciones hospitalarias durante 2017 (al año siguiente). Notas: *Ajustado por edad y sexo. E: desvío estandar; IC 95\%: intervalo de confianza del $95 \%$; RI: Razón de incidencia (en inglés Incidence Rate Ratio). NA: no aplica (valor de referencia)

\begin{tabular}{|l|c|c|c|c|}
\hline Quintilo & $\begin{array}{c}\text { Internaciones por } \\
\text { quintilo de UPCa, Media } \\
\text { (DE) }\end{array}$ & $\begin{array}{c}\text { Internaciones por } \\
\text { quintilo de COCa, } \\
\text { Media (DE) }\end{array}$ & UPCa, RI (IC 95 \%) $)^{*}$ & COCa, RI (IC 95 \%)* \\
\hline 1 & $0,13(0,70)$ & $0,12(0,70)$ & NA & NA \\
2 & $0,12(0,52)$ & $0,12(0,51)$ & $0,83(0,76$ a 0,92$)$ & $0,84(0,76$ a 0,93$)$ \\
3 & $0,11(0,50)$ & $0,11(0,52)$ & $0,78(0,71$ a 0,87$)$ & $0,81(0,73$ a 0,91$)$ \\
4 & $0,13(0,52)$ & $0,13(0,52)$ & $0,83(0,75$ a 0,91$)$ & $0,83(0,75$ a 0,92$)$ \\
5 & $0,09(0,45)$ & $0,09(0,45)$ & $0,62(0,56$ a 0,69$)$ & $0,63(0,57$ a 0,70$)$ \\
\hline
\end{tabular}

Recibido el 08/10/2020 Aceptado el 10/03/2021 y Publicado el 21/04/2021.

\section{Agradecimientos}

A la Dra. Mónica Gabriela Schpilberg, la Lic. Adriana lannicelli y la Ing. Mariana Landoni, por sus inestimables esfuerzos en la búsqueda y recuperación de los datos administrativos y la posterior construcción de los indicadores; y al Dr Ignacio R. Ricci por sus valiosos aportes durante la revisión de pares que permitieron mejorar el manuscrito.

\section{Referencias}

1. da Cunha EM, Giovanella L. Longitudinalidade/continuidade do cuidado: identificando dimensões e variáveis para a avaliação da Atenção Primária no contexto do sistema público de saúde brasileiro. Ciênc saúde coletiva. 2011;16(suppl 1):1029-1042. Available from: 10.1590/S141381232011000700036;https://www.scielo.br/scielo.php?script=sci_arttext\&pid=S1413-81232011000700036.

2. Haggerty JL, Reid RJ, Freeman GK, et al. Continuity of care: a multidisciplinary review. BMJ. 2003;327(7425):1219-1221. Available from: 10.1136/ bmj.327.7425.1219.

3. Meiqari L, Al-Oudat T, Essink D, et al. How have researchers defined and used the concept of 'continuity of care' for chronic conditions in the context of resource-constrained settings? A scoping review of existing literature and a proposed conceptual framework. Health Res Policy Syst. 2019;17(1):27. Available from: 10.1186/s12961-019-0426-1.

4. G V. Continuidad del cuidado. Evid Actual Pract Ambul. 2021;24(01):e002112. Available from: http://www.evidencia.org.ar/index.php/Evidencia/ article/view/6922.

5. Introducción a la práctica de la Medicina Familiar. In: Rubinstein E, et al., editors. PROFAM, Salud del Anciano. Buenos Aires: Fundación MF Para el desarrollo de la Medicina Familiar y la Atención primaria de la salud; 2006.

6. Reid RJ, Haggerty JL, McKendry R. Defusing the confusion: Concepts and measures of continuity of healthcare; 2002. Available from: https://www.semanticscholar.org/paper/Defusing-the-Confusion\%3A-Concepts-and-Measures-of-of-Reid-Haggerty/ 468205b4dab997d9148208f601f9ac938b396154.

7. Cabana MD, Jee SH. Does continuity of care improve patient outcomes? J Fam Pract. 2004;53(12):974-80.

8. Saultz JW, Lochner J. Interpersonal Continuity of Care and Care Outcomes: A Critical Review. Ann Fam Med. 2005;3(2):159-166. Available from: 10.1370/afm.285.

9. van Loenen T, van-den Berg MJ, Westert GP, et al. Organizational aspects of primary care related to avoidable hospitalization: a systematic review Fam Pract. 2014;31(5):502-516. Available from: 10.1093/fampra/cmu053.

10. Puntis S, Rugkåsa J, Forrest A, et al. Associations between continuity of care and patient outcomes in mental health care: a systematic review. Psychiatr Serv. 2015;66(4):354-363. Available from: 10.1176/appi.ps.201400178.

11. Yang F, Xiong ZF, Yang C, et al. Continuity of Care to Prevent Readmissions for Patients with Chronic Obstructive Pulmonary Disease: A Systematic Review and Meta-Analysis. COPD. 2017;14(2):251-261. Available from: 10.1080/15412555.2016.1256384.

12. Gray DJP, Sidaway-Lee K, White E, Thorne A, Evans PH. Continuity of care with doctors-a matter of life and death? A systematic review of continuity of care and mortality. BMJ Open. 2018 June;8(6):e021161. Available from: 10.1136/bmjopen-2017-021161.

13. D'haenens F, Rompaey BV, Swinnen E, et al. The effects of continuity of care on the health of mother and child in the postnatal period: a systematic review. European Journal of Public Health. 2020;30(4):749-760. Available from: 10.1093/eurpub/ckz082.

14. Baker R, Freeman GK, Haggerty JL, Bankart MJ, Nockels KH. Primary medical care continuity and patient mortality: a systematic review. British Journal of General Practice. 2020 August;Available from: 10.3399/bjgp20X712289. 
15. Plan de Salud del Hospital Italiano de Buenos Aires;. Available from: https://www.hospitalitaliano.org.ar/\#!/home/plan/inicial [Last access: 24/03/2021].

16. Rubinstein A, Rubinstein F, Botargues M, et al. A Multimodal Strategy Based on Pay-Per-Performance to Improve Quality of Care of Family Practitioners in Argentina. J Ambul Care Manage. 2009;32(2):103-114. Available from: 10.1097/JAC.0b013e31819940f7.

17. Saultz JW. Defining and Measuring Interpersonal Continuity of Care. Ann Fam Med. 2003;1(3):134-143. Available from: 10.1370/afm.23.

18. Jee SH, Cabana MD. Indices for continuity of care: a systematic review of the literature. Med Care Res Rev. 2006;63(2):158-188. Available from: $10.1177 / 1077558705285294$

19. Bentler SE, Morgan RO, Virnig BA, et al. The Association of Longitudinal and Interpersonal Continuity of Care with Emergency Department Use, Hospitalization, and Mortality among Medicare Beneficiaries. PLoS ONE. 2014;9(12):e115088. Available from: 10.1371/journal.pone.0115088.

20. Pourat N, Davis AC, Chen X, et al. In California, Primary Care Continuity Was Associated With Reduced Emergency Department Use And Fewer Hospitalizations. Health Aff (Millwood). 2015;34(7):1113-1120. Available from: 10.1377/hlthaff.2014.1165.

21. Barker I, Steventon A, Deeny SR. Association between continuity of care in general practice and hospital admissions for ambulatory care sensitive conditions: Cross sectional study of routinely collected, person level data. BMJ (Online). 2017;356:j84. Available from: 10.1136/bmj.j84.

22. Dreiher J, Comaneshter DS, Rosenbluth Y, et al. The association between continuity of care in the community and health outcomes: a populationbased study. Isr J Health Policy Res. 2012;1(1):21. Available from: 10.1186/2045-4015-1-21.

23. Kohnke $\mathrm{H}$, Zielinski A. Association between continuity of care in Swedish primary care and emergency services utilisation: a population-based cross-sectional study. Scand J Prim Health Care. 2017;35(2):113-119. Available from: 10.1080/02813432.2017.1333303.

24. Leleu H, Minvielle E. Relationship between Longitudinal Continuity of Primary Care and Likelihood of Death: Analysis of National Insurance Data. PLoS ONE. 2013 August;8(8):e71669. Available from: 10.1371/journal.pone.0071669. 